\title{
Article
}

\section{Comparison of Laboratory and Daily-Life Gait Speed Assessment during ON and OFF States in Parkinson's Disease}

\author{
Marta Francisca Corrà ${ }^{1,2, *}$, Arash Atrsaei $\left.{ }^{3}{ }^{(}\right)$, Ana Sardoreira ${ }^{2}$, Clint Hansen ${ }^{4}\left(\mathbb{D}\right.$, Kamiar Aminian ${ }^{3}(\mathbb{D}$, \\ Manuel Correia ${ }^{1,2}$, Nuno Vila-Chã ${ }^{2}$, Walter Maetzler ${ }^{4}$ and Luís Maia ${ }^{1,2,5}$ \\ 1 Abel Salazar Biomedical Sciences Institute (ICBAS), University of Porto, 4050-313 Porto, Portugal; \\ mcorreia.neurologia@chporto.min-saude.pt (M.C.); luismaia.neurologia@chporto.min-saude.pt (L.M.) \\ 2 University Hospital Santo Antonio of Porto (CHUP), 4099-001 Porto, Portugal; \\ ana.sardoeira@gmail.com (A.S.); nunovilacha@hotmail.com (N.V.-C.) \\ 3 Laboratory of Movement Analysis and Measurement, Swiss Federal Institute of Technology in \\ Lausanne (EPFL), 1015 Lausanne, Switzerland; atrsaei.arash@epfl.ch (A.A.); kamiar.aminian@epfl.ch (K.A.) \\ 4 Department of Neurology, Christian-Albrechts-University, 24118 Kiel, Germany; \\ c.hansen@neurologie.uni-kiel.de (C.H.); w.maetzler@neurologie.uni-kiel.de (W.M.) \\ 5 Institute for Research and Innovation in Health (i3s), University of Porto, 4200-135 Porto, Portugal \\ * Correspondence: martafrancisca.corra@gmail.com
}

\section{check for} updates

Citation: Corrà, M.F.; Atrsaei, A.; Sardoreira, A.; Hansen, C.; Aminian, K.; Correia, M.; Vila-Chã, N.;

Maetzler, W.; Maia, L. Comparison of Laboratory and Daily-Life Gait Speed Assessment during ON and OFF States in Parkinson's Disease. Sensors 2021, 21, 3974. https://doi.org/ $10.3390 /$ s21123974

Academic Editor: Emmanouil Rousakis

Received: 28 April 2021

Accepted: 7 June 2021

Published: 9 June 2021

Publisher's Note: MDPI stays neutral with regard to jurisdictional claims in published maps and institutional affiliations.

Copyright: (c) 2021 by the authors. Licensee MDPI, Basel, Switzerland. This article is an open access article distributed under the terms and conditions of the Creative Commons Attribution (CC BY) license (https:/ / creativecommons.org/licenses/by/ $4.0 /)$.

\begin{abstract}
Accurate assessment of Parkinson's disease (PD) ON and OFF states in the usual environment is essential for tailoring optimal treatments. Wearables facilitate measurements of gait in novel and unsupervised environments; however, differences between unsupervised and in-laboratory measures have been reported in PD. We aimed to investigate whether unsupervised gait speed discriminates medication states and which supervised tests most accurately represent home performance. In-lab gait speeds from different gait tasks were compared to home speeds of 27 PD patients at $\mathrm{ON}$ and OFF states using inertial sensors. Daily gait speed distribution was expressed in percentiles and walking bout (WB) length. Gait speeds differentiated $\mathrm{ON}$ and OFF states in the lab and the home. When comparing lab with home performance, $\mathrm{ON}$ assessments in the lab showed moderate-to-high correlations with faster gait speeds in unsupervised environment $(r=0.69 ; p<0.001)$, associated with long WB. OFF gait assessments in the lab showed moderate correlation values with slow gait speeds during OFF state at home $(r=0.56 ; p=0.004)$, associated with short WB. In-lab and daily assessments of gait speed with wearables capture additional integrative aspects of PD, reflecting different aspects of mobility. Unsupervised assessment using wearables adds complementary information to the clinical assessment of motor fluctuations in PD.
\end{abstract}

Keywords: remote patient monitoring; medication states; Parkinson's disease; lab vs. home; wearable sensors; human gait; gait speed

\section{Introduction}

Parkinson's disease (PD) is a chronic and progressive neurodegenerative disorder characterized by impairment of mobility and gait, with severe consequences on quality of life. Motor symptoms and gait impairment are mainly caused by loss of dopaminergic neurons in the substantia nigra, decreasing dopamine levels in the brain [1]. Thus, current treatments of PD focus on increasing dopamine delivery: among the main dopaminergic medications, levodopa is considered the gold standard therapy [2]. Up to $50 \%$ of PD patients within two years of levodopa therapy may begin to experience mild motor fluctuations [3]. Motor fluctuations represent alternations of periods of good dopaminergic effect, with adequate control of movements (the perception of this state by the affected patient is called "ON state") to others of poor control and significant worsening of motor symptoms (comparably, this perception by the affected patient is called "OFF state") [4].

Reduction of motor fluctuations is an important indicator to evaluate the effectiveness of pharmacological interventions. The most popular tool to quantitatively assess motor 
fluctuations is the motor part of the Unified Parkinson's Disease Rating Scale (UPDRS, and the Movement Disorder Society-revised version thereof), a validated clinical rating scale of PD symptoms that includes both historical information and clinical examination for $\mathrm{ON}$ and OFF states [5]. However, clinical scores do not necessarily provide representative data on the patient's daily performance at home. An alternative method of tracking motor fluctuations is to ask patients to fill a diary differentiating various symptoms during the day, and to rate and define their current status of being at ON state or at a decreased medication effect by self-perception. This method has several limitations, including recall bias, reduced compliance, and the need to be accurately compiled to have valid and interpretable data [6]. In addition, these methods do not involve quantitative measures of movements. Monitoring objective gait parameters to track motor fluctuations may compensate these subjective limitations, and allow more sensitivity, accuracy and reproducibility [7].

More recently, wearable health technologies have been developed with the possibility to investigate PD symptoms at a new level of granularity and in novel environments that have previously not been covered by clinical evaluations [8]. In particular, inertial measurement units (IMUs) are electronic devices worn on the body that can detect movements and successfully estimate spatial-temporal parameters, using a combination of accelerometers, gyroscopes and sometimes magnetometers. Thanks to the reduced size and costs of their components, they are easy to wear and low-cost tools for movement analysis [8]. Compared to more complex equipment such as 3D optical motion capture systems, IMUs are less time consuming and do not require specific expertise to use. In addition, complex tools are used only in clinical settings due to their high cost and complexity of technology, and do not often represent the full gait complexity [9]. The use of inertial measurement units (IMUs) indeed makes it now possible to investigate motor features such as gait and motor performance in unsupervised conditions such as the domestic environment. This may enable a passive collection of clinically important information, such as durations of medication ON and OFF states, in natural environments of individuals [10-12]. Among the parameters extracted from IMUs, gait speed was shown to be one of the most reliable predictors of mobility [13], risk of falling [14,15], and loss of independence [16], as well as a powerful indicator of changes in performances over time [17]. Gait speed is a critical measure of gait function for different pathologies $[13,18,19]$. Stratification of gait activity in the home environment in walking bouts (WBs) of different lengths seems to provide additional useful insight into mobility performance [20].

It has been observed that gait speed can significantly differ when analyzed at home or in the laboratory $[13,19,21-24]$. Carcreff et al. [13] showed lower gait speed values in daily life compared to lab in a group of children with cerebral palsy. In this study, children were asked to walk barefoot during the lab assessment, which may generate great differences when comparing both assessments. Two other studies $[19,23]$ have compared supervised and unsupervised gait speeds in the elderly. A weak association between daily-life- and labobtained gait speed was found by Takayanagi et al. [19], with average daily gait speed being significantly lower than lab speed. De La Camara et al. [23] showed an association with speed and physical, mental and cognitive health outcomes, and highlighted that clinically obtained gait speed can underestimate or overestimate habitual gait speed. All of these studies used IMUs to detect mobility, but they all presented limitations regarding the type of gait tasks used for the lab assessment. For example, distance walked in the lab was between 2.44 and $10 \mathrm{~m}$, which can be considered too short to be compared with daily gait speed. Even in PD, it has been shown that supervised instruments to measure motor symptoms do not strongly reflect daily-living activity [21]. For example, no significant correlation was observed between lab and home gait parameters in a study by Toosizadeh et al. [22], but the small sample size and methodological differences (one single sensor on the sternum) between lab and home assessments of the study may have affected the accuracy of the results. Therefore, it is still unclear whether supervised and unsupervised assessments are as sensitive when used to identify motor fluctuations in PD. Precise information on the degree of association between supervised and unsupervised assessments of motor 
fluctuations is still lacking. Improving the understanding of gait disabilities with additional information about daily-life performances from IMUs is essential to enhance clinical care, design personalized interventions and overcome limitations concerning questionnaires and self-reported diaries.

For these reasons, in the present study, we compared gait speed from supervised (in the laboratory) and unsupervised (at home and daily-life conditions) assessments to determine the degree of association of the different medication states in PD patients. In particular, we tested whether gait speed in unsupervised environments discriminates ON and OFF states, and investigated which supervised tests most accurately represent home performance during both medication states, also in relation to clinical scores.

\section{Materials and Methods}

\subsection{Patients}

PD patients diagnosed by a movement disorder specialist based on the UK Brain Bank criteria [25] were recruited. The following exclusion criteria were applied:

- $\quad$ Older than 90 years of age;

- Dementia;

- $\quad$ Any relevant gait-impairing health issue other than PD;

- $\quad$ Unable to walk a distance of $20 \mathrm{~m}$;

- $\quad$ Not taking anti-parkinsonian medications in the past month;

- $\quad$ Difference of less than 2 points between ON and OFF in the UPDRS-III, in order to consider the minimum clinically significant difference between states [26,27].

Demographic data and information on medication intake were collected [28]. The main characteristics of the population are presented in the results section. All subjects gave their informed consent for inclusion before they participated in the study. The study was conducted in accordance with the Declaration of Helsinki, and the protocol was approved by the Ethics Committee of Centro Hospitalar Universitário do Porto (CHUP) with identification code 2018.087(076-DEFI/076-CES).

\subsection{Data Collection}

During the first day of assessment, participants were first evaluated with UPDRS part III (a medical professional evaluates actual motor performance) in their OFF state in the morning, at least $12 \mathrm{~h}$ after the last dose of dopaminergic medication intake. They were then equipped with two synchronized RehaGait IMUs (Hasomed GmbH, Magdeburg, Germany), each containing a tri-axial gyroscope and tri-axial accelerometer (Figure 1). These sensors were located on both feet, recording data with a sampling frequency of $100 \mathrm{~Hz}$. Participants were asked to perform the following gait tasks: a 20-m straight walking test at normal pace and at fast pace; and a circular walking task, which is walking at normal pace three times around a circular carpet of $1.2 \mathrm{~m}$ diameter, at both left and right directions (Figure 1). Then, participants were asked to take their usual first dose of dopaminergic medication, and the same assessment (UPDRS scores and gait assessment) was performed between 1 and $2 \mathrm{~h}$ after intake of medication.

During the second day of assessment, participants wore a Physilog ${ }^{\circledR} 5$ IMU (Gait Up, Lausanne, Switzerland) on the right foot between 9:00 and 9:30 a.m. (Figure 1). The device comprises a triaxial accelerometer and gyroscope with a sampling frequency of $128 \mathrm{~Hz}$. For the following $12 \mathrm{~h}$ (during a weekday), participants were then asked to keep wearing the IMUs and perform usual daily activities in their domestic environment, including their usual outdoor activities. For convenience, the terms 'home' or 'domestic environment' will include outdoor activities during the day of the assessment.

Both inertial sensors used in the study showed the same technical characteristics and measure the same type of data, guaranteeing no differences in terms of results and set-ups (Figure 1). In addition, participants were asked to fill in a diary over the day to report the times of dopaminergic medication intake. To ensure a precise documentation of time and 
quantity of dopaminergic medication intake, caregivers were also instructed to monitor the diary record.

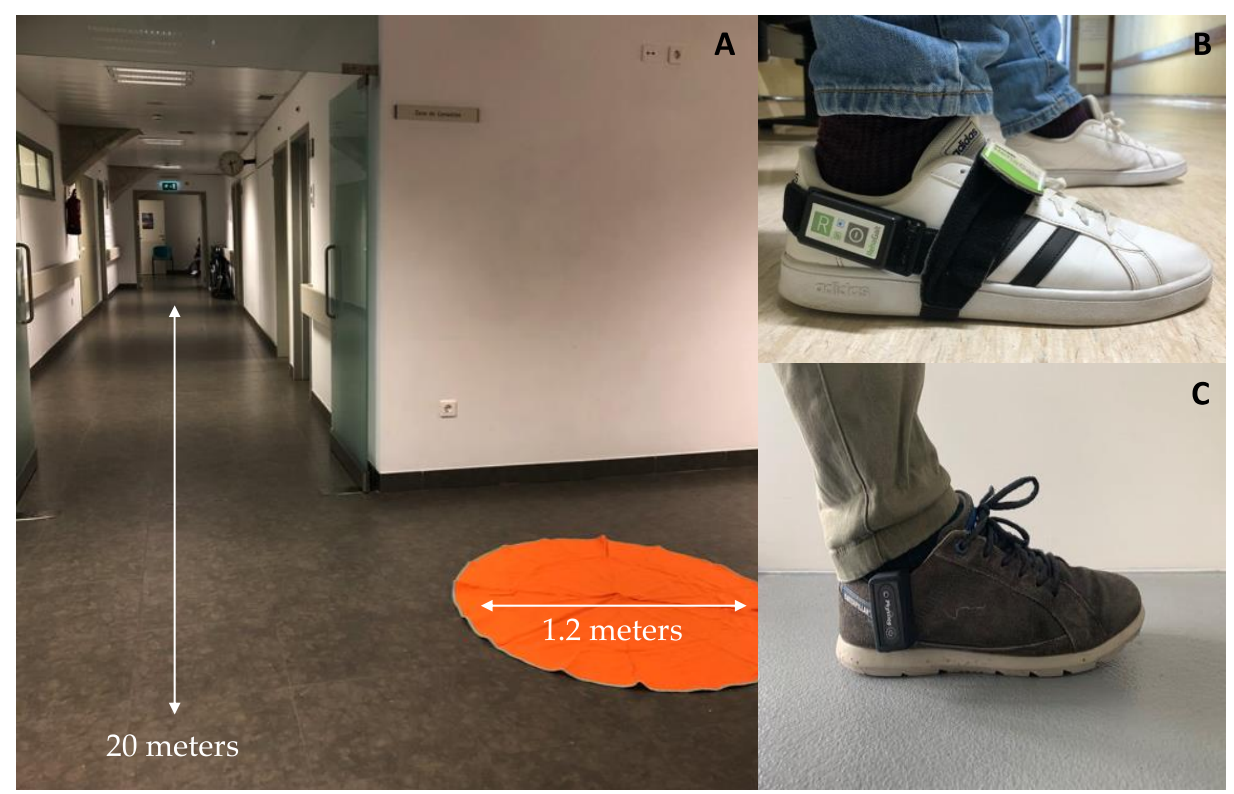

Figure 1. (A) Lab setting for the supervised assessment: $20 \mathrm{~m}$ walking and circular walking tasks. (B). Sensor positioning for lab assessment. (C). Sensor positioning for unsupervised assessment.

\subsection{Data Processing and Extracted Parameters}

Raw data were processed using Matlab R2020b (MathWorks, Nantick, MA, USA). To analyze gait speeds, the raw data of the IMU from the right foot was used for both supervised and unsupervised assessments. To ensure no systematic biases between assessments in terms of heterogeneity, usage and accuracy, raw data of accelerometer and gyroscope from the two assessments were processed with the same algorithm to obtain gait speed [29]. This algorithm had been validated in a previous study on PD patients and achieved an accuracy ( \pm precision) of $2.8( \pm 2.4) \mathrm{cm} / \mathrm{s}$ [30].

From the lab assessment, gait speed was calculated for all walking tests (20-m walk test at normal and fast pace, and the circular walking test). Two strides at the beginning and end of the tests, respectively, were excluded to obtain steady-state gait speed values.

As described in the reference [29], the raw accelerometer and gyroscope data were processed to first detect the gait events. The acceleration of the movement was integrated to obtain the velocity of the foot. During the motionless periods, the zero-velocity update approach was applied to overcome the drift problem. Gait speed was then calculated from the drift-free velocity. Details of the procedure are provided in [29]. An example of extracted data is shown in Figure 2.

From the home assessment, due to the complex context of the unsupervised setting, and the vast distribution of gait speed [24], two approaches were employed. In the first approach, gait speed was obtained from each stride. In the second approach, mean gait speed per walking bout (WB) was calculated. WBs were determined as described earlier [31], and then divided into short (15-30 s), medium (>30-60 s) and long WBs (>60 s). WBs shorter than $15 \mathrm{~s}$ were not included to avoid any influence on the accuracy of the used algorithm. Gait cycles having less than $0.2 \mathrm{~m} / \mathrm{s}$ of gait speed were not considered, as they can be assumed to be static periods. Gait bouts were then allocated to respective ON and not-ON states: ON state was arbitrarily defined as 60-180 min after dopaminergic medication intake (based on the dopaminergic intake time in the diary). The period between $30 \mathrm{~min}$ before and $30 \mathrm{~min}$ after dopaminergic medication intake was defined as not-ON state, describing the condition in which no optimal drug effect is to be assumed [32-34]. 


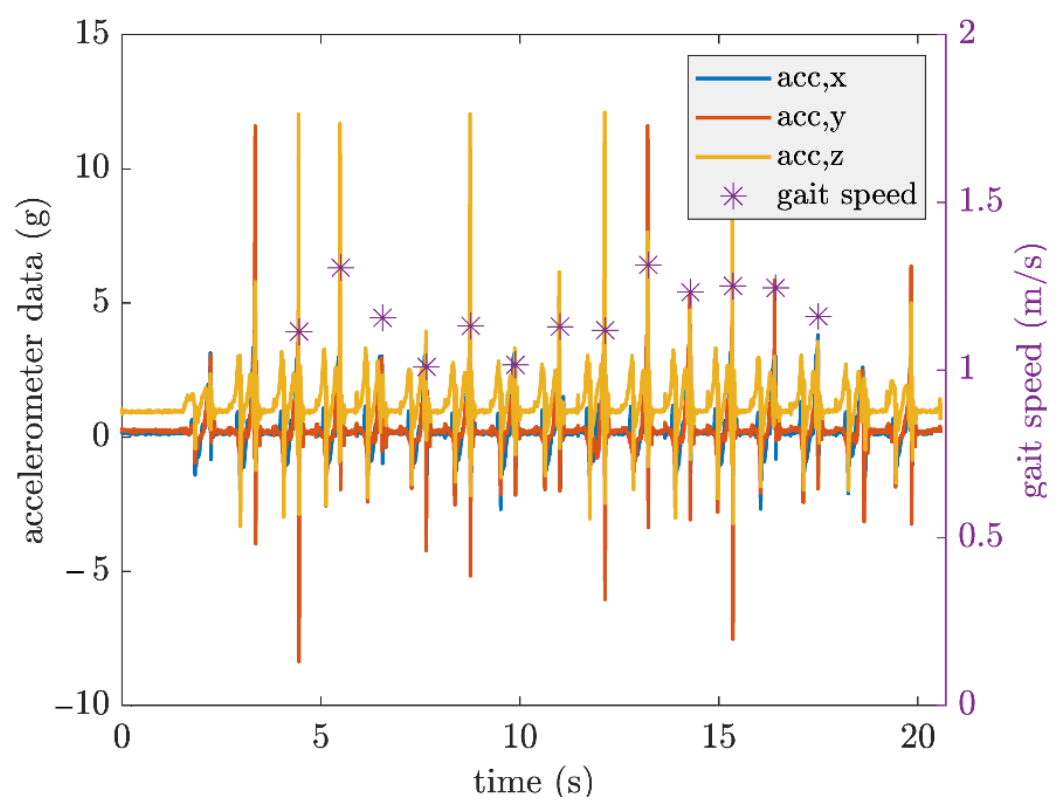

Figure 2. Example of gait speed extraction from a PD patient during straight walking at fast pace. Gait speed is given for each gait cycle of the right foot.

\subsection{Statistical Analysis}

From the lab data, mean gait speeds of each trial were extracted. From home-based data, the 25th, 50th, 75th, 90th percentiles and maximum values of gait speeds were calculated from all gait cycles. The use of different percentiles was based on previous studies showing a heterogeneous distribution of daily-life gait speed in the elderly, resulting in relevant correlations with higher percentiles with capacity in the lab [35].

From the WB approach, we calculated the mean gait speed within each WB type, and the corresponding 25th, 50th and maximum values of each WBs type were considered for both $\mathrm{ON}$ and not-ON medication states [35].

Normality of data was checked with Shapiro-Wilk test. To compare different medication states in the lab $(\mathrm{ON}, \mathrm{OFF})$ and home environment $(\mathrm{ON}$, not-ON), a paired comparison ( $t$-test for parametric data; Wilcoxon signed rank test for non-parametric data) was used, and $p$ values $<0.05$ were considered significant.

In order to compare PD patients' gait speed at home and in the lab at both medication states, respectively, and in relation to clinical scores, we performed a correlation analysis (Pearson correlation for parametric data; Spearman correlation for non-parametric data). A correlation coefficient of less than 0.5 was considered as low, between 0.5 and 0.7 as moderate and above 0.7 as high [36]. In addition, to measure the proportion of the variance of home assessment that is predictable from the lab assessments, the coefficient of determination $\left(R^{2}\right)$ was calculated, applying data transformation for non-normally distributed variables. All statistical analysis was performed using IBM $^{\circledR}$ SPSS 25 package.

\section{Results}

\subsection{Demographic and Clinical Characteristics}

A total of 39 PD patients were initially recruited. Out of this group, a total of 27 patients ( $40.7 \%$ female) met the inclusion criteria and performed the entire study protocol. Included participants did not significantly differ in demographic and clinical characteristics from those not included (Supplementary Table S1). Median age of the included participants was 69 years and the median disease duration was six years. Seventeen PD patients $(63 \%)$ were early stage (Hoehn and Yahr stage $\leq 2$ during medication OFF state), and 10 patients (37\%) at mild-to-moderate stage (Hoehn and Yahrstage 2.5-3). Demographic and clinical details are provided in Table 1. 
Table 1. Demographics and clinical characteristics of PD patients.

\begin{tabular}{cc}
\hline Variables & Value (IQR) \\
\hline Male: Female & $16: 11$ \\
Age (years) & $69(64-73)$ \\
Disease duration (years) & $6(3-9)$ \\
Disease onset (years) & $64(57-69)$ \\
Hoehn and Yahr stage (0-5) & ON: 2 \\
UPDRS I (0-16) & OFF: 2 \\
UPDRS II (0-52) & $2(1-4)$ \\
UPDRS III (0-108) & $7(3-10)$ \\
UPDRS IV (0-23) & ON: $12(8-20)$ \\
Total LED (mg) & OFF: $22(15-31)$ \\
\hline
\end{tabular}

Note: Results are expressed in median and interquartile range (IQR). LED: Levodopa equivalent dose; UPDRS Unified Parkinson's Disease Rating Scale.

\subsection{Gait Speed}

\subsubsection{Comparison of Gait Speeds between Respective ON and OFF/not-ON States}

Relative gait speeds in the lab and at home are shown in Figure 3. In the lab, straight walking at normal pace had a median value of $1.01 \mathrm{~m} / \mathrm{s}$ during the medication ON state, and $0.97 \mathrm{~m} / \mathrm{s}$ during the medication OFF state $(p=0.004)$. During circular walking it reached $0.69 \mathrm{~m} / \mathrm{s}$ during the $\mathrm{ON}$, and $0.58 \mathrm{~m} / \mathrm{s}$ during the OFF state $(p<0.001)$. Only two PD patients were able to perform the straight walking at fast pace assessment during the medication OFF state; therefore, this task was not included in the analysis.

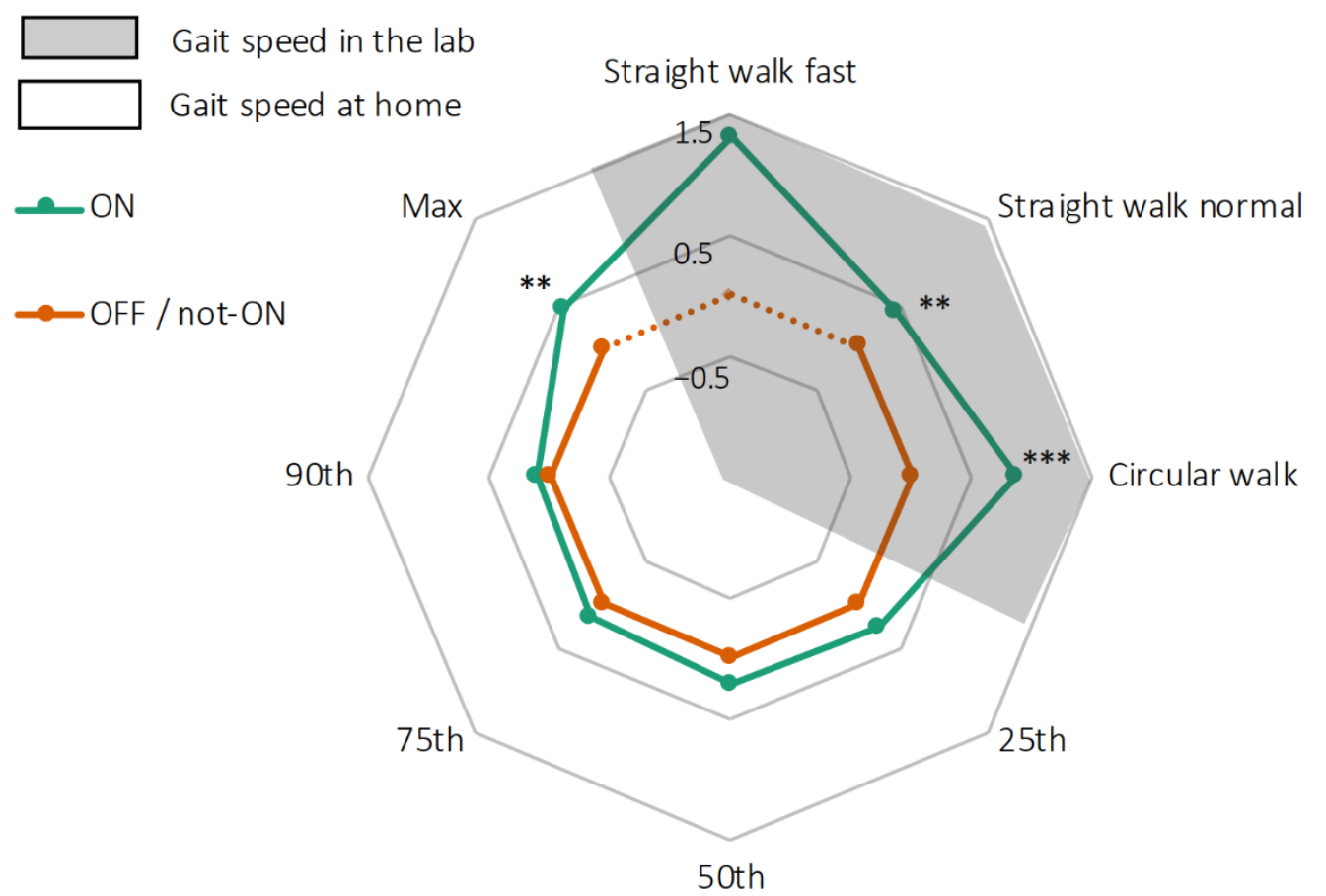

Figure 3. Radar plot illustrating gait speeds of $27 \mathrm{PD}$ patients during lab tests (in grey) and in their domestic environment (white, presented by different percentiles). Gait speeds during medication $\mathrm{OFF}$ in the lab and not-ON in the domestic environment are presented as 0 (orange line), and gait speeds during medication $\mathrm{ON}$ are presented as deviation from 0 (green line). Straight walking test at fast pace was not performed during OFF medication state (dotted orange line). ${ }^{* *} p<0.01$, *** $p<0.001$.

In the domestic environment, the median gait speed was $0.83 \mathrm{~m} / \mathrm{s}$ during the medication $\mathrm{ON}$ state and $0.77 \mathrm{~m} / \mathrm{s}$ during the medication not-ON state $(p=0.302)$. A significant 
difference was found between $\mathrm{ON}$ and not-ON medication states for the maximum gait speed $(\mathrm{ON}=1.48 \mathrm{~m} / \mathrm{s}$; not- $\mathrm{ON}=1.36 \mathrm{~m} / \mathrm{s} ; p=0.009)$, but not for the other percentiles (Figure 3).

3.2.2. Comparison of Gait Speeds between Lab and Home Environment during Medication ON State

During the medication ON states, low correlations were found when comparing gait speeds obtained from the normal walking tasks in the lab with the maximum values of home gait speed $(r=0.46 ; p=0.02)$. Moderate correlations of gait speeds were observed between the fast walking task in the lab and the 90th percentile $(r=0.64 ; p<0.001)$ and maximum values $(r=0.69 ; p<0.001)$ of the home-derived data. Similar results were found when comparing the circular walking task in the lab with the 90th percentile $(\mathrm{r}=0.53$; $p=0.004)$ and maximum values $(r=0.61 ; p=0.001)$. In general, the degrees of correlation between lab and home gait speeds increased with higher percentiles of gait speed in the unsupervised environment. This was also reflected by the $\mathrm{R}^{2}$ values (Table 2; Figure S1).

Table 2. Correlation of $\mathrm{ON}$ and $\mathrm{OFF} /$ not-ON state between the lab and the domestic environment.

ON

\begin{tabular}{|c|c|c|c|c|c|c|c|c|c|}
\hline & \multicolumn{3}{|c|}{ Straight Walking Fast Pace } & \multicolumn{3}{|c|}{ Straight Walking Normal Pace } & \multicolumn{3}{|c|}{ Circular Walking } \\
\hline & Comparison & Correlation & \multirow{2}{*}{$R^{2}(\%)$} & Comparison & Correlation & \multirow{2}{*}{$R^{2}(\%)$} & Comparison & Correlation & \multirow{2}{*}{$R^{2}(\%)$} \\
\hline & $p$-Value & $r$ & & $p$-Value & $r$ & & $p$-Value & $r$ & \\
\hline 25 th & $<0.001$ & $0.45 *$ & 20 & $<0.001$ & $0.38 *$ & 14 & 0.715 & 0.36 & 13 \\
\hline 50 th & $<0.001$ & $0.54^{* *}$ & 29 & 0.003 & $0.40 *$ & 16 & $<0.001$ & $0.49 * *$ & 25 \\
\hline 75th & $<0.001$ & $0.60 * *$ & 37 & 0.495 & $0.40 *$ & 16 & $<0.001$ & $0.53 * *$ & 28 \\
\hline 90th & $<0.001$ & $0.64^{* * *}$ & 41 & 0.132 & 0.40 * & 16 & $<0.001$ & $0.53 * *$ & 28 \\
\hline Max & $<0.001$ & $0.69^{* * *}$ & 30 & $<0.001$ & $0.46^{*}$ & 15 & $<0.001$ & $0.61 * *$ & 39 \\
\hline \multicolumn{10}{|c|}{ OFF/not-ON } \\
\hline & \multicolumn{3}{|c|}{ Straight Walking Fast Pace } & \multicolumn{3}{|c|}{ Straight Walking Normal Pace } & \multicolumn{3}{|c|}{ Circular Walking } \\
\hline & Comparison & Correlation & \multirow{2}{*}{$R^{2}(\%)$} & Comparison & Correlation & \multirow{2}{*}{$R^{2}(\%)$} & Comparison & Correlation & \multirow{2}{*}{$\mathbf{R}^{2}(\%)$} \\
\hline & $p$-Value & $r$ & & $p$-Value & $r$ & & $p$-Value & $r$ & \\
\hline 25 th & \multirow{5}{*}{\multicolumn{3}{|c|}{$\begin{array}{c}\text { Straight walking test at fast pace was } \\
\text { not performed during OFF } \\
\text { medication state. }\end{array}$}} & $<0.001$ & $0.56^{* *}$ & 33 & 0.038 & $0.55^{* *}$ & 31 \\
\hline 50 th & & & & 0.009 & $0.42 *$ & 18 & $<0.001$ & $0.39 *$ & 16 \\
\hline 75th & & & & 0.893 & 0.36 & 14 & $<0.001$ & $0.38 *$ & 15 \\
\hline 90th & & & & 0.030 & 0.34 & 12 & $<0.001$ & 0.41 * & 17 \\
\hline Max & & & & $<0.001$ & 0.15 & 2 & $<0.001$ & 0.37 & 14 \\
\hline
\end{tabular}

Paired comparisons ( $p$-value), degrees of correlation $(r)$ and coefficients of determination $\left(\mathrm{R}^{2}\right)$ between lab tests and most relevant percentiles of gait speed in the domestic environment during $\mathrm{ON}$ and $\mathrm{OFF} /$ not-ON medication state. Correlation asterisks represent the following $p$-values: ${ }^{*} p<0.05,{ }^{* *} p<0.01,{ }^{* * *} p<0.001$.

3.2.3. Comparison of Gait Speeds between Lab and Home Environment during Medication $\mathrm{OFF} /$ not-ON State

During the medication OFF/not-ON states, moderate correlations were found when comparing gait speeds obtained from the normal walking tasks in the lab with the 25th percentile of home gait speed $(r=0.56 ; p=0.004)$. Similar results were also found when comparing gait speed of the circular walking task in the lab with the 25th percentile of gait speed as obtained from the home data $(r=0.55 ; p=0.004)$. This was also reflected by the $\mathrm{R}^{2}$ values (Table 2; Figure $\mathrm{S} 1$ ).

3.2.4. Comparison of Gait Speeds between Lab and Home Environment during Opposite Medication States

Low correlations were found when comparing gait speeds obtained from the normal walking task in the lab at $\mathrm{ON}$ state with the maximum values of home gait speed at not-ON $(r=0.26 ; p=0.209)$. Moderate correlations of gait speeds were observed between the 
fast walking task in the lab at $\mathrm{ON}$ and the maximum values of the home-derived data at not-ON $(r=0.47 ; p=0.015)$. Similar results were found when comparing the circular walking task in the lab at ON with maximum value of home gait speed at not-ON $(r=0.41$; $p=0.035$ ) (Supplementary Table S2). The same lower percentiles were observed between measurements at OFF in the lab and $\mathrm{ON}$ in domestic environment: gait speed from the normal walking tasks at OFF and the 25th percentile from home at $\mathrm{ON}(r=0.38 ; p=0.063)$; circular walking task at OFF and the 25th percentile from home at $\mathrm{ON}(r=0.43 ; p=0.031)$.

\subsection{Comparison of Gait Speeds in the Home Environment, Stratified by Different Bout Lengths}

In the home environment, WBs of different lengths most probably reflect different purposes of walking, such as doing the housework (short WBs) and taking a walk (long WBs) [37]. We found no significant differences in the number of WBs between $\mathrm{ON}$ and not-ON medication states (Supplementary Table S3). When comparing gait speeds between $\mathrm{ON}$ and not-ON states, stratified by different WB lengths, we found significant differences between $\mathrm{ON}$ and not-ON medication states only at high gait speeds. Moreover, this was only observed in short and medium WBs (short WBs: $p=0.026$; medium WBs: $p=0.008$ ). Long WBs did not add relevant information (Figure 4).

Short WB at home

Medium WB at home

Long WB at home

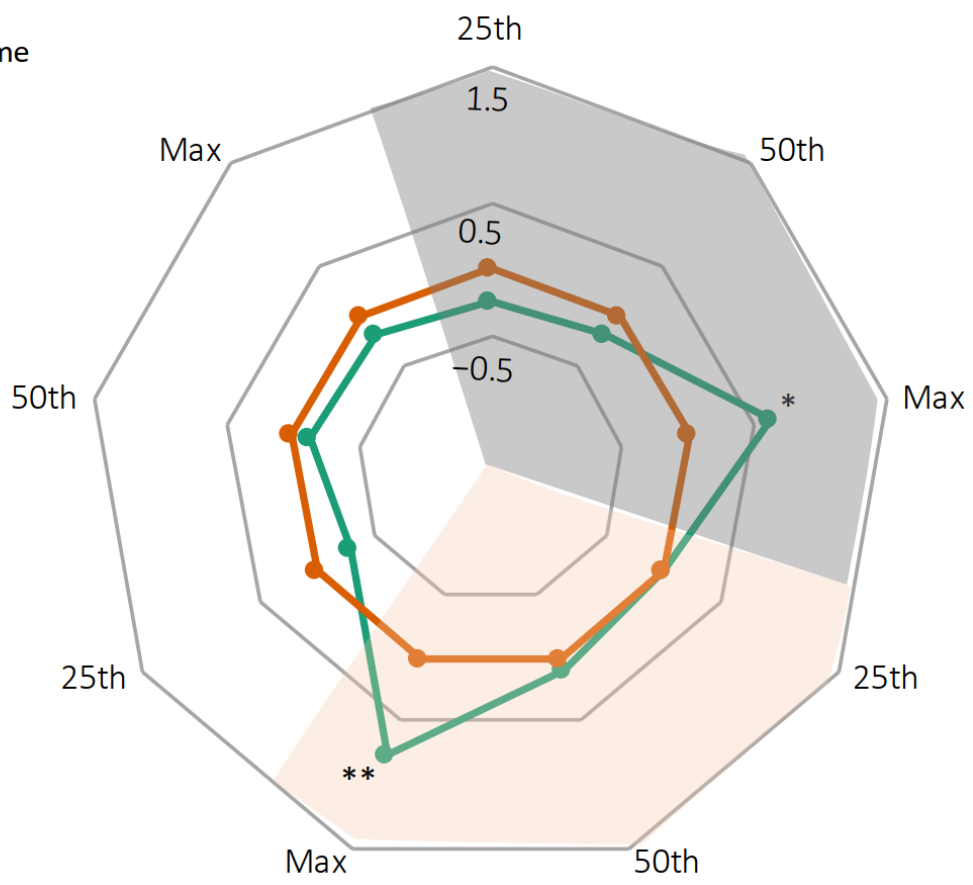

Figure 4. Radar plot illustrating gait speeds of $27 \mathrm{PD}$ patients in their domestic environment, broken down into different WB. Gait speeds during medication not-ON are set at 0 (orange line), gait speeds during medication $\mathrm{ON}$ are presented as deviation from 0 (green line). ${ }^{*} p<0.05,{ }^{* *} p<0.01$.

We then compared gait speeds from the lab with those obtained from the domestic environment during ON state, stratified by WB lengths. Degrees of correlation were highest between the fast walking task in the lab and the maximum gait speed during long WBs $(r=0.63 ; p<0.001)$ and the circular walking task in the lab and the 50th percentile of gait speed during short WBs $(r=0.72 ; p<0.001)$ (Table 3; Figure S2). 
Table 3. Correlation of $\mathrm{ON}$ and $\mathrm{OFF} /$ not-ON medication states between the lab and the domestic environment according to WB.

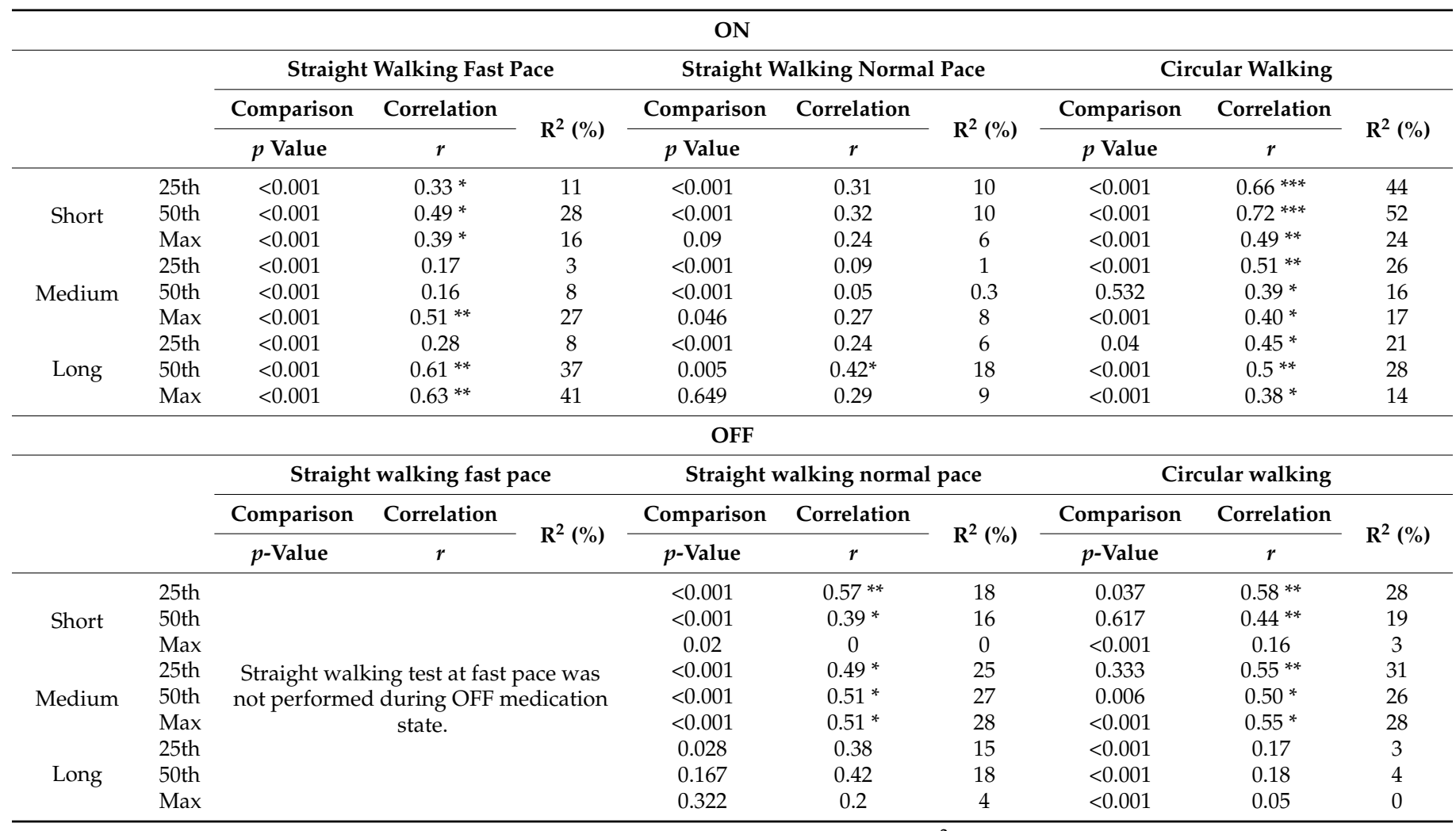

Paired comparisons ( $p$-value), degrees of correlation $(r)$ and coefficients of determination $\left(\mathrm{R}^{2}\right)$ between lab tests and WB of gait speed in the domestic environment during ON and OFF/not-ON medication state. Correlation asterisks represent the following $p$-values: ${ }^{*} p<0.05$, ${ }^{* *} p<0.01,{ }^{* * *} p<0.001$.

Consistently, when comparing gait speed between the lab and the domestic environment during OFF/not-ON state, stratified by WB lengths, degrees of correlation were highest between the normal walking task in the lab and the 25th percentile of gait speed during short WBs $(r=0.57 ; p=0.004)$, and the circular walking task in the lab and the 25th percentile of gait speed during short WBs $(r=0.58 ; p=0.003)$ (Table 3; Figure S2).

\subsection{Comparison of Home-Collected Gait Speeds with UPDRS-III Scores}

At ON state, there were no significant correlations between the UPDRS-III ON scores and the home-collected gait speed percentiles. Only item 30, assessing gait, significantly correlated with the 90th percentile of home-collected gait speed $(r=-0.61 ; p=0.001)$.

At not-ON state, there were no significant correlations between the UPDRS-III OFF scores and the home-collected gait speed percentiles at not-OFF. Only item 30 moderately correlated with the 25 th percentile of home-collected gait speed $(r=-0.44 ; p=0.028)$.

\section{Discussion}

This exploratory cross-sectional study with PD patients during medication $\mathrm{ON}$ and $\mathrm{OFF}$ /not-ON states investigates gait speeds obtained from the lab and from a home assessment.

Firstly, gait speed was the only objective parameter considered in this analysis. Due to its combination of temporal and spatial gait characteristics, it is the most reliable predictor of mobility, and a valid and easy-to-administer measure of walking, which can reliably be estimated using IMUs [13].

Secondly, we decided to use different gait tasks in the lab and different set-ups in order to gather inertial signals similar to those that would be obtained in the daily life of PD patients. Other studies have only compared daily-life gait speed with tasks of short 
distances at normal pace, making this comparison less reliable $[13,19,22,23]$. We confirmed the importance of using both lab and home assessments to add considerable explanatory value to the understanding of PD motor function.

We found relevant differences in maximum gait speeds in the home environment between medication $\mathrm{ON}$ and not-ON states (Figure 1). When analyzing the best performance at home (i.e., the maximum values of gait speed at home) we were able to discriminate medication states. Moreover, when we analyzed gait speeds stratified by WB lengths, we found that maximum values of gait speed during short and medium WBs provided more informative to discriminate $\mathrm{ON}$ and not-ON states in PD. This finding suggests that maximum values of gait speed at home better represent the maximum capacity in the lab rather than normal daily-life performance. This may help clinicians to have a more precise estimation of the patient's capacity when measuring mobility in unsupervised conditions. Considering extreme values of gait speed may be more informative when measuring mobility and the patient's motor status. Furthermore, shorter walking bouts provide more discriminative information compared to longer walking bouts as short walking bouts might be accompanied by other cognitive or motor tasks. Therefore, we recommend considering these parameters as useful indicators when evaluating PD treatment's effect in daily-life environments $[22,24,37]$. In unsupervised settings, the segmentation of WBs by length (short and medium WBs) provided additional information on individual patient $\mathrm{ON} /$ not-ON state.

We report, to the best of our knowledge, for the first time, that specific percentiles of gait speed and WB lengths may help in differentiating and monitoring ON and not-ON states in PD. Previous studies aimed to differentiate PD states in the home environment using other parameters rather than gait [38], or through the development of algorithms and machine learning approaches for quantifying specific PD motor symptoms, such as tremor, bradykinesia and dyskinesia [39-42]. Our study adds to such studies by including unsupervised gait speed performance as a relevant parameter to accurately monitor PD patients with mobile health technology, ultimately aiming at personalized adjustments in PD therapy.

We found that gait speed as assessed in medication ON state in the lab reflects (i) gait speeds obtained in the home during $\mathrm{ON}$ are better than during not-ON states, (ii) faster speeds correlated higher than slower speeds, and (iii) high correlations were mainly obtained in the long WBs. In more detail, we found the strongest association of fast pace in the lab with the highest gait speed percentiles $(r=0.69 ; p<0.001)$ and long WBs $(0.63$; $p=0.002$ ) of daily gait speed. This supports the concept that maximum capacity in the lab can efficiently reflect the best performance in daily life, and that assessments of capacity are possible in both lab and home environments.

In contrast, daily gait speed showed only low correlation with walking at preferred pace in the lab $(r=0.46)$. This lab gait task may thus not reliably reflect the complexity of mobility at $\mathrm{ON}$ state in everyday life, probably because asking to walk at 'considered normal' speed may lead to different interpretations, and cause a less homogeneous speed. Therefore, assessing fast speed in the lab may give more information on motor functions during daily-life activities. These discrepancies are also in line with previous studies: no significant correlation was found between walking at normal pace in the lab and home assessment [22], and lab-based gait assessment explained less than one third of the daily-living activity in PD [21]. This may be related to an increased awareness when performing tasks in a supervised environment or because of limited ecological validity when performing isolated movements [21,22,24]. Therefore, assessing fast speed in the lab may give more information on motor functions during daily-life activities.

We also observed that circular walking in the lab can moderately represent a patient's everyday gait speed at home $(r=0.61)$. This association was pronounced in short WBs, which may reflect more complex and demanding gait situations in daily life, such as specific activities including walking and acting with the hands simultaneously [37,43]. Evidence from literature supports this hypothesis: gait features obtained in unsupervised 
conditions were closer to gait features obtained in the lab during dual-tasking, than when only walking [44-47].

In contrast, gait speed as assessed in medication OFF state in the lab reflects (i) gait speeds obtained in the home during not-ON are better than during ON states, (ii) slower speeds correlated higher than faster speeds, and (iii) high correlations were mainly obtained in the short WBs. In more detail, gait speed obtained during OFF state in the lab seems to best reflect how PD patients perform at home when below their usual performance, confirmed by the association of home-collected gait speed of lower percentiles (25th) and short WBs with all the lab tests $(r=0.56 ; p=0.004)$. This is contrary to the results observed during respective $\mathrm{ON}$ states, where the higher was gait speed in the domestic environment, the higher was the correlation value with fast gait speed in the lab. Nevertheless, for this specific dataset, less information was obtained during not-ON state.

Taken together, these findings show that, during medication ON states, respectively, the fast speed gait value obtained in the lab can nicely inform about how PD patients perform their fast walking activities in their usual environment, and, similarly, circular walking can provide information about how PD patients behave in relation to more complex everyday tasks. By contrast, during medication OFF/not-ON states, the slow gait speed value as obtained in the lab can well inform about how PD patients perform their slow walking activities in their usual environment. We could also show a high discriminant validity of these findings because the respective opposite correlations did not show significant results (Supplementary Table S2).

These results are of clinical relevance as they suggest that straight walking at preferred speed in the lab may be substituted by alternative measurements if the aim is to collect information relevant for everyday life of $\mathrm{PD}$ patients at $\mathrm{ON}$ state. However, even if the most suitable assessment is performed, only about $40 \%$ of home gait speed could be explained by the lab assessment. This means that lab tests generally explain less than half of the home performance and, consequently, monitoring of daily-life gait seems to be of utmost relevance for a comprehensive understanding of PD gait in usual environments [21].

We also compared gait speed from real-life settings with the UPDRS measurements, which are based on a one-time physical examination. Only specific items of the UPDRS-III showed significant correlations with PD gait performance in everyday life. Comparably similar results were observed in a previous study [48], where no significant correlation was found among the entire UPDRS subgroups, but gait features showed significant correlations with specific items of the UPDRS-III, mostly related to gait. Therefore, it could be an advantage to assess PD mobility using objective and targeted parameters from lab and home-based tools.

The present study has some limitations. First, the sample size was relatively small. Second, unsupervised assessments were performed for only one day. We based this choice on the following considerations: a previous study [49] evaluated the repeatability of sensorbased assessments during two consecutive days and found that gait parameters were highly comparable between the two days. In addition, home and lab measures demonstrated strong discriminatory power in detecting impaired motor function in PD in another study, where unsupervised assessment was performed during one single day [22]. Still, further studies with several days of measurements are needed to capture a more granular picture of daily mobility [13]. Third, $\mathrm{ON}$ and not-ON states during daily-life activities were defined based on diary entries. Future studies should focus on a set-up that includes clearly defined OFF states (e.g., mornings, before medication intake).

We did not consider WBs shorter than $15 \mathrm{~s}$, because we aimed to analyze steady-state gait and compare an equal number of steps with the lab capacity. However, in everyday situations, there are many shorter WBs occurring within the home or in-door conditions $(<10$ s) $[11,37]$. Since daily-living gait often takes place by using very short WBs, such bouts should also be considered in future analyses. Finally, the intra-subject variability of gait speed was considered only in the calculation of gait speed percentiles and not directly from 
the increment and decrement for each subject. Since it could be an interesting biomarker for the investigation of PD mobility, such analysis should be considered in further studies.

\section{Conclusions}

In-lab and daily-living testing with wearables can capture complementary aspects of PD, and substantially add to clinical evaluation and patient management. We highlighted which specific laboratory tests can better represent gait speed at home. This can be useful for clinicians, since it is possible to remotely assess the capacity of the patients in their domestic environment, and protect more vulnerable people, especially during the COVID-19 pandemic. On the other hand, if clinicians decide to perform only supervised tests, they know which functional tests are more indicative of patients' daily-life performance. Another relevant highlight is the importance of including two different methods (percentiles of the total gait distribution and WB types) in the analysis of home-collected gait speed, for a more detailed representation of PD daily-life performance.

Improving the understanding of gait disabilities with additional information about daily-life performances from IMUs could enhance clinical care, design personalized interventions and overcome limitations concerning questionnaires and self-reported diaries.

Supplementary Materials: The following are available online at https:/ /www.mdpi.com/article/10 $.3390 / \mathrm{s} 21123974 / \mathrm{s} 1$, Figure S1: Correlation of ON and OFF/not-ON state between the lab and the most relevant percentiles of domestic environment, Figure S2: Correlation of ON and OFF/not-ON state between the lab and the most relevant WBs of domestic environment, Table S1: Demographic data of the patients included and excluded in the analysis, Table S2: Correlation of ON and OFF /not-ON states between the lab and the domestic environment, Table S3: Characteristics of WB during $\mathrm{ON}$ and $\mathrm{OFF} /$ not-ON medication states.

Author Contributions: Conceptualization, M.F.C., A.A., L.M. and W.M.; methodology, A.S., N.V.C., M.F.C., A.A.; formal analysis, M.F.C.; data curation, A.A. and C.H.; writing-original draft preparation, M.F.C.; writing—review and editing, M.F.C., A.A., A.S., N.V.-C., M.C., K.A., C.H., L.M. and W.M.; visualization, M.C.; supervision, L.M., K.A., W.M., M.C.; project administration, L.M., M.C.; funding acquisition, M.F.C., A.A. All authors have read and agreed to the published version of the manuscript.

Funding: This research was funded by Keep Control from the EU's Horizon 2020 (H2020) research and innovation program under the Marie Sklodowska-Curie (MSCA-ITN-ETN), grant number 721577. No other financial support and funding for the preceding twelve months are applied.

Institutional Review Board Statement: The study was conducted according to the guidelines of the Declaration of Helsinki, and approved by the Institutional Review Board (or Ethics Committee) of Centro Hospitalar Universitario do Porto (protocol code 2018.087(076-DEFI/076-CES) 10 May 2018).

Informed Consent Statement: Informed consent was obtained from all subjects involved in the study.

Data Availability Statement: The data presented in this study are available on request from the corresponding author.

Acknowledgments: We thank Paula Salgado, Margarida Calejo and Ana Luisa Sousa for their help in data collection, and Benoit Mariani for his assistance with the wearable sensors.

Conflicts of Interest: The authors declare no conflict of interest.

\section{References}

1. Lotharius, J.; Brundin, P. Pathogenesis of parkinson's disease: Dopamine, vesicles and $\alpha$-synuclein. Nat. Rev. Neurosci. 2002, 3, 932-942. [CrossRef]

2. Poewe, W.; Antonini, A.; Zijlmans, J.C.; Burkhard, P.R.; Vingerhoets, F. Levodopa in the treatment of Parkinson's disease: An old drug still going strong. Clin. Interv. Aging 2010, 5, 229-238.

3. Olanow, C.W.; Kieburtz, K.; Rascol, O.; Poewe, W.; Schapira, A.H.; Emre, M.; Nissinen, H.; Leinonen, M.; Stocchi, F. Factors predictive of the development of Levodopa-induced dyskinesia and wearing-off in Parkinson's disease. Mov. Disord. 2013, 28, 1064-1071. [CrossRef] 
4. Borzì, L.; Varrecchia, M.; Olmo, G.; Artusi, C.A.; Fabbri, M.; Rizzone, M.G.; Romagnolo, A.; Zibetti, M.; Lopiano, L. Home monitoring of motor fluctuations in Parkinson's disease patients. J. Reliab. Intell. Environ. 2019, 5, 145-162. [CrossRef]

5. Rodríguez-Molinero, A.; Samà, A.; Pérez-López, C.; Rodríguez-Martín, D.; Quinlan, L.R.; Alcaine, S.; Mestre, B.; Quispe, P.; Giuliani, B.; Vainstein, G.; et al. Analysis of Correlation between an Accelerometer-Based Algorithm for Detecting Parkinsonian Gait and UPDRS Subscales. Front. Neurol. 2017, 8, 431. [CrossRef]

6. Reimer, J.; Grabowski, M.; Lindvall, O.; Hagell, P. Use and interpretation of on/off diaries in Parkinson's disease. J. Neurol. Neurosurg. Psychiatry 2004, 75, 396-400. [CrossRef]

7. Godinho, C.; Domingos, J.; Cunha, G.; Santos, A.T.; Fernandes, R.M.; Abreu, D.; Gonçalves, N.; Matthews, H.; Isaacs, T.; Duffen, J.; et al. A systematic review of the characteristics and validity of monitoring technologies to assess Parkinson's disease. J. Neuroeng. Rehabil. 2016, 13, 24. [CrossRef] [PubMed]

8. Brognara, L.; Palumbo, P.; Grimm, B.; Palmerini, L. Assessing Gait in Parkinson's Disease Using Wearable Motion Sensors: A Systematic Review. Diseases 2019, 7, 18. [CrossRef]

9. Ridao-Fernández, C.; Pinero-Pinto, E.; Chamorro-Moriana, G. Observational Gait Assessment Scales in Patients with Walking Disorders: Systematic Review. BioMed Res. Int. 2019, 2019, 2085039. [CrossRef] [PubMed]

10. Rovini, E.; Maremmani, C.; Cavallo, F. How Wearable Sensors Can Support Parkinson's Disease Diagnosis and Treatment: A Systematic Review. Front. Neurosci. 2017, 11, 555. [CrossRef] [PubMed]

11. Del Din, S.; Godfrey, A.; Mazza, C.; Lord, S.; Rochester, L. Free-living monitoring of Parkinson's disease: Lessons from the field. Mov. Disord. 2016, 31, 1293-1313. [CrossRef]

12. Hssayeni, M.D.; Burack, M.A.; Jimenez-Shahed, J.; Ghoraani, B. Assessment of response to medication in individuals with Parkinson's disease. Med. Eng. Phys. 2019, 67, 33-43. [CrossRef]

13. Carcreff, L.; Gerber, C.N.; Paraschiv-Ionescu, A.; De Coulon, G.; Aminian, K.; Newman, C.J.; Armand, S. Walking Speed of Children and Adolescents With Cerebral Palsy: Laboratory Versus Daily Life. Front. Bioeng. Biotechnol. 2020, 8, 812. [CrossRef]

14. Paker, N.; Bugdayci, D.; Goksenoglu, G.; Demircioğlu, D.T.; Kesiktas, N.; Ince, N. Gait speed and related factors in Parkinson's disease. J. Phys. Ther. Sci. 2015, 27, 3675-3679. [CrossRef] [PubMed]

15. Creaby, M.W.; Cole, M.H. Gait characteristics and falls in Parkinson's disease: A systematic review and meta-analysis. Parkinsonism Relat. Disord. 2018, 57, 1-8. [CrossRef] [PubMed]

16. Bryant, M.S.; Rintala, D.H.; Hou, J.G.; Charness, A.L.; Fernandez, A.L.; Collins, R.L.; Baker, J.; Lai, E.C.; Protas, E.J. Gait variability in Parkinson's disease: Influence of walking speed and dopaminergic treatment. Neurol. Res. 2011, 33, 959-964. [CrossRef] [PubMed]

17. Erb, M.K.; Karlin, D.R.; Ho, B.K.; Thomas, K.C.; Parisi, F.; Vergara-Diaz, G.P.; Daneault, J.-F.; Wacnik, P.W.; Zhang, H.; Kangarloo, T.; et al. mHealth and wearable technology should replace motor diaries to track motor fluctuations in Parkinson's disease. Npj Digit. Med. 2020, 3, 6. [CrossRef]

18. Carcreff, L.; Gerber, C.N.; Paraschiv-Ionescu, A.; De Coulon, G.; Newman, C.J.; Aminian, K.; Armand, S. Comparison of gait characteristics between clinical and daily life settings in children with cerebral palsy. Sci. Rep. 2020, 10, 2091. [CrossRef]

19. Takayanagi, N.; Sudo, M.; Yamashiro, Y.; Lee, S.; Kobayashi, Y.; Niki, Y.; Shimada, H. Relationship between Daily and In-laboratory Gait Speed among Healthy Community-dwelling Older Adults. Sci. Rep. 2019, 9, 3496. [CrossRef]

20. Hass, C.J.; Bishop, M.; Moscovich, M.; Stegemöller, E.L.; Skinner, J.; Malaty, I.A.; Shukla, A.W.; McFarland, N.; Okun, M.S. Defining the Clinically Meaningful Difference in Gait Speed in Persons With Parkinson Disease. J. Neurol. Phys. Ther. 2014, 38, 233-238. [CrossRef]

21. Galperin, I.; Hillel, I.; Del Din, S.; Bekkers, E.M.J.; Nieuwboer, A.; Abbruzzese, G.; Avanzino, L.; Nieuwhof, F.; Bloem, B.R.; Rochester, L.; et al. Associations between daily-living physical activity and laboratory-based assessments of motor severity in patients with falls and Parkinson's disease. Parkinsonism Relat. Disord. 2019, 62, 85-90. [CrossRef]

22. Toosizadeh, N.; Mohler, J.; Lei, H.; Parvaneh, S.; Sherman, S.; Najafi, B. Motor Performance Assessment in Parkinson's Disease: Association between Objective In-Clinic, Objective In-Home, and Subjective/Semi-Objective Measures. PLoS ONE 2015, 10, e0124763. [CrossRef] [PubMed]

23. De la Cámara, M.; Higueras-Fresnillo, S.; Sadarangani, K.P.; Esteban-Cornejo, I.; Martinez-Gomez, D.; Veiga, Ó.L. Clinical and Ambulatory Gait Speed in Older Adults: Associations With Several Physical, Mental, and Cognitive Health Outcomes. Phys. Ther 2020, 100, 718-727. [CrossRef] [PubMed]

24. Warmerdam, E.; Hausdorff, J.M.; Atrsaei, A.; Zhou, Y.; Mirelman, A.; Aminian, K.; Espay, A.J.; Hansen, C.; Evers, L.J.W.; Keller, A.; et al. Long-term unsupervised mobility assessment in movement disorders. Lancet Neurol. 2020, 19, 462-470. [CrossRef]

25. Hughes, A.J.; Daniel, S.E.; Kilford, L.; Lees, A.J. Accuracy of clinical diagnosis of idiopathic Parkinson's disease: A clinicopathological study of 100 cases. J. Neurol. Neurosurg. Psychiatry 1992, 55, 181-184. [CrossRef]

26. Shulman, L.M.; Gruber-Baldini, A.L.; Anderson, K.E.; Fishman, P.S.; Reich, S.G.; Weiner, W.J. The clinically important difference on the unified Parkinson's disease rating scale. Arch. Neurol. 2010, 67, 64-70. [CrossRef]

27. Elshehabi, M.; Maier, K.S.; Hasmann, S.E.; Nussbaum, S.; Herbst, H.; Heger, T.; Berg, D.; Hobert, M.A.; Maetzler, W. Limited Effect of Dopaminergic Medication on Straight Walking and Turning in Early-to-Moderate Parkinson's Disease during Single and Dual Tasking. Front. Aging Neurosci. 2016, 8, 4. [CrossRef] [PubMed]

28. Tomlinson, C.L.; Stowe, R.; Patel, S.; Rick, C.; Gray, R.; Clarke, C.E. Systematic review of levodopa dose equivalency reporting in Parkinson's disease. Mov. Disord. 2010, 25, 2649-2653. [CrossRef] 
29. Mariani, B.; Hoskovec, C.; Rochat, S.; Büla, C.; Penders, J.; Aminian, K. 3D gait assessment in young and elderly subjects using foot-worn inertial sensors. J. Biomech. 2010, 43, 2999-3006. [CrossRef]

30. Mariani, B.; Jiménez, M.C.; Vingerhoets, F.J.G.; Aminian, K. On-Shoe Wearable Sensors for Gait and Turning Assessment of Patients With Parkinson's Disease. IEEE Trans. Biomed. Eng. 2013, 60, 155-158. [CrossRef]

31. El Achkar, C.M.; Lenoble-Hoskovec, C.; Paraschiv-Ionescu, A.; Major, K.; Büla, C.; Aminian, K. Instrumented shoes for activity classification in the elderly. Gait Posture 2016, 44, 12-17. [CrossRef]

32. Rastgardani, T.; Armstrong, M.J.; Gagliardi, A.R.; Marras, C. Understanding, Impact, and Communication of "Off" Periods in Parkinson's Disease: A Scoping Review. Mov. Disord. Clin. Pract. 2018, 5, 461-470. [CrossRef] [PubMed]

33. Nelson, M.V.; Berchou, R.C.; Lewitt, P.A.; Kareti, D.; Kesaree, N.; Schlick, P.; Galloway, M.P. Pharmacokinetic and pharmacodynamic modeling of L-dopa plasma concentrations and clinical effects in Parkinson's disease after Sinemet. Clin. Neuropharmacol. 1989, 12, 91-97. [CrossRef] [PubMed]

34. Bordelon, Y.M.; Hays, R.D.; Vassar, S.D.; Diaz, N.; Bronstein, J.; Vickrey, B.G. Medication responsiveness of motor symptoms in a population-based study of Parkinson disease. Parkinsons Dis. 2011, 2011, 967839. [CrossRef] [PubMed]

35. Van Ancum, J.M.; van Schooten, K.S.; Jonkman, N.H.; Huijben, B.; van Lummel, R.C.; Meskers, C.G.M.; Maier, A.B.; Pijnappels, M. Gait speed assessed by a 4-m walk test is not representative of daily-life gait speed in community-dwelling adults. Maturitas 2019, 121, 28-34. [CrossRef] [PubMed]

36. Van Lummel, R.C.; Walgaard, S.; Pijnappels, M.; Elders, P.J.; Garcia-Aymerich, J.; van Dieën, J.H.; Beek, P.J. Physical Performance and Physical Activity in Older Adults: Associated but Separate Domains of Physical Function in Old Age. PLoS ONE 2015, 10, e0144048. [CrossRef]

37. Del Din, S.; Godfrey, A.; Galna, B.; Lord, S.; Rochester, L. Free-living gait characteristics in ageing and Parkinson's disease: Impact of environment and ambulatory bout length. J. Neuroeng. Rehabil. 2016, 13, 46. [CrossRef]

38. Moore, S.T.; MacDougall, H.G.; Gracies, J.M.; Cohen, H.S.; Ondo, W.G. Long-term monitoring of gait in Parkinson's disease. Gait Posture 2007, 26, 200-207. [CrossRef]

39. Pulliam, C.L.; Heldman, D.A.; Brokaw, E.B.; Mera, T.O.; Mari, Z.K.; Burack, M.A. Continuous Assessment of Levodopa Response in Parkinson's Disease Using Wearable Motion Sensors. IEEE Trans. Biomed. Eng. 2018, 65, 159-164. [CrossRef] [PubMed]

40. Pérez-López, C.; Samà, A.; Rodríguez-Martín, D.; Moreno-Aróstegui, J.M.; Cabestany, J.; Bayes, A.; Mestre, B.; Alcaine, S.; Quispe, P.; Laighin, G.; et al. Dopaminergic-induced dyskinesia assessment based on a single belt-worn accelerometer. Artif. Intell. Med. 2016, 67, 47-56. [CrossRef]

41. Lonini, L.; Dai, A.; Shawen, N.; Simuni, T.; Poon, C.; Shimanovich, L.; Daeschler, M.; Ghaffari, R.; Rogers, J.A.; Jayaraman, A. Wearable sensors for Parkinson's disease: Which data are worth collecting for training symptom detection models. NPJ Digit. Med. 2018, 1, 64. [CrossRef] [PubMed]

42. Aich, S.; Youn, J.; Chakraborty, S.; Pradhan, P.M.; Park, J.H.; Park, S.; Park, J. A Supervised Machine Learning Approach to Detect the On/Off State in Parkinson's Disease Using Wearable Based Gait Signals. Diagnostics 2020, 10, 421. [CrossRef] [PubMed]

43. Shah, V.V.M.J.; Harker, G.; Mancini, M.; Carlson-Kuhta, P.; Nutt, J.G.; El-Gohary, M.; Curtze, C.; Horak, F.B. Effect of Bout Length on Gait Measures in People with and without Parkinson's Disease during Daily Life. Sensors 2020, 20, 5769. [CrossRef]

44. Hillel, I.; Gazit, E.; Nieuwboer, A.; Avanzino, L.; Rochester, L.; Cereatti, A.; Croce, U.D.; Rikkert, M.O.; Bloem, B.R.; Pelosin, E.; et al. Is every-day walking in older adults more analogous to dual-task walking or to usual walking? Elucidating the gaps between gait performance in the lab and during 24/7 monitoring. Eur. Rev. Aging Phys. Act. 2019, 16, 6. [CrossRef]

45. Toulotte, C.; Thevenon, A.; Watelain, E.; Fabre, C. Identification of healthy elderly fallers and non-fallers by gait analysis under dual-task conditions. Clin. Rehabil 2006, 20, 269-276. [CrossRef] [PubMed]

46. Digo, E.; Agostini, V.; Pastorelli, S.; Gastaldi, L.; Panero, E. Gait Phases Detection in Elderly Using Trunk-MIMU System; SciTePress: Setúbal, Portugal, 2021; pp. 58-65.

47. Montero-Odasso, M.; Casas, A.; Hansen, K.T.; Bilski, P.; Gutmanis, I.; Wells, J.L.; Borrie, M.J. Quantitative gait analysis under dual-task in older people with mild cognitive impairment: A reliability study. J. Neuroeng. Rehabil. 2009, 6, 35. [CrossRef]

48. Weiss, A.; Sharifi, S.; Plotnik, M.; van Vugt, J.P.; Giladi, N.; Hausdorff, J.M. Toward automated, at-home assessment of mobility among patients with Parkinson disease, using a body-worn accelerometer. Neurorehabilit. Neural Repair 2011, 25, 810-818. [CrossRef] [PubMed]

49. Kumar, D.P.; Wendel, C.; Mohler, J.; Laksari, K.; Toosizadeh, N. Between-day repeatability of sensor-based in-home gait assessment among older adults: Assessing the effect of frailty. Aging Clin. Exp. Res. 2020. [CrossRef] 